\title{
A Design of Experiments Approach to Investigating the Sensitivity of the Re-order Point Method
}

\author{
Peter Nielsen ${ }^{1}$, Giovanni Davoli ${ }^{2}$, Izabela Nielsen ${ }^{1}$, and Niels Gorm Maly Rytter ${ }^{1}$ \\ ${ }^{1}$ Department of Mechanical and Manufacturing Engineering, Aalborg University, \\ Fibigerstraede 16, 9220 Aalborg Oest, Denmark \\ \{peter, Izabela, i9nr\}@m-tech.aau.dk \\ ${ }^{2}$ Department of Mechanical and Civil Engineering (DIMeC), University of Modena and \\ Reggio Emilia; via Vignolese 905, 41100, Modena, Italy \\ giovanni.davoli@unimore.it
}

\begin{abstract}
This paper investigates the re-order point inventory management models sensitivity to demand distributions, demand dependencies and lead time distributions. The investigated performance measures are four different versions of service level. The conclusion is for all measures that the single most critical aspect adversely affecting service level performance is the presence of asymmetrically distributed demand.
\end{abstract}

Keywords: Inventory management, discrete event simulation, design of experiment, demand distributions.

\section{Introduction}

Inventory management is a practical challenge faced by many companies across industries. Inventory management can be reduced to the single objective of maintaining the cost optimal amount of inventory on hand. However, in practice this is typically reduced to achieving a certain service level i.e. satisfying a certain percentage of demand directly from inventory. The correct service level will be situational, some schools e.g. advocated in Silver et al. (1998), that the service level is a direct trade off between various cost factors (typically holding vs. cost of stock out). Other would argue that it is determined by market requirements. Regardless of how the target service level is determined, it is possible to calculate for a given situation the amount of safety stock needed to achieve the target.

This paper presents the results from an investigation of the behavior of a simple reorder point inventory management method. The aim is to determine which factors, settings and the combination of factors influence on the achieved service level. For this purpose a simulation study is carried out using XCV software. The study is structured around a design of experiments and investigates four different service level measures and their sensitivity to four different parameters: demand distribution, correlation of demand, mean lead time and standard deviation of lead time. The aim of the study is to 1) identify which factors and settings of these that are critical regardless of 
service level measure 2) provide insight into the complexity of real life inventory management by identifying which factors interact 3) give guidelines for how to improve service level measures in practice.

The remainder of the paper is structured as follows. First, a literature review of inventory management, demand distributions and simulation studies of inventory management is presented. This is followed by a design of experiments. Third, results from a full factorial experiment are presented and discussed before final conclusions and avenues of further research are presented.

\section{$2 \quad$ Literature Review}

In general most methods used in practice for inventory management tend to be based on assumptions of normally independently distributed lead time demand (e.g. Silver et al. (1998), Vollmann et al. (2005). For many practical purposes this will also tend to be a reasonable assumption. However, Tadikamalla (1984) concludes that high values of Coefficient of Variance associated with asymmetrical distributions of demand lead to poor performance of inventory management techniques and that the symmetry/asymmetry of demand is critical for e.g. inventory costs (Zotteri, 2000). Bobko and Whybark (1985) find that the coefficient of variation is in fact a robust descriptor of the degree lumpiness. Tadikamalla (1984) likewise underlines that it is necessary to deviate from the assumptions of normality / symmetrical distributed (lead time) demand to achieve a satisfactory performance. Zotteri (2000) finds that the shape of the demand distribution is critical when determining the performance of inventory management methods. However, other methods (e.g. aggregation in time (Eriksen and Nielsen, 2011)) tend to be able to compensate for the asymmetry experienced. This could also indicate that there is a critical level of asymmetry of demand distribution (especially when taking into account a given lead time) that when reached will lead to poor performance. Within inventory management individual customer orders are translated into a demand rate (with a given distribution) and used for calculating reorder points, lot-sizes, timing, safety stock etc. (Silver, 1981). This is often translated into a lead time demand distribution. The shorter the lead time period, the closer the lead time demand distribution is to the actual distribution of demand. It however seems necessary to investigate to which extend inventory management techniques are sensitive to these and other parameters.

Inventory management is can be fundamentally be reduced to a combined decision of how much and when to order. In most instances this problem is reduced to taking into account (for a given cost structure; holding, ordering, stock out) four factors:

- Demand distribution $\left(\mathrm{D}_{\mathrm{d}}\right)$ (sometimes modeled as lead time demand, in this paper modeled as the more complex daily demand)

- Correlated demand $\left(\mathrm{C}_{\mathrm{d}}\right)$

- Mean lead time $\left(\mu_{\mathrm{LT}}\right)$

- Standard deviation of lead time $\left(\sigma_{\mathrm{LT}}\right)$ 
To investigate these factors this paper utilizes the same methods as the ones presented in e.g. Bobko and Whybark (1985) and Zotteri (2000).

\section{Design of Experiments}

To investigate the behavior of the re-order point method a simulation experiment is completed. The aim of the experiment is to investigate the importance of the four factors on the service level performance of the ROP. The aim is not to develop a predictive response model. A full factorial experiment with three levels is used in this paper. Four factors and three levels give $3^{4}=81$ combinations and thus 81 separate experiments were conducted. The three settings for the four factors are shown in Table 1.

Table 1. Overview of experimental settings of the four investigated factors

\begin{tabular}{llll}
\hline & Low (-1) & Mean (0) & High (+1) \\
\hline$D_{d}$ & $\begin{array}{l}\text { Normal distribution, mean }= \\
\text { 1.000 units/day, standard } \\
\text { deviation = 300 units/day }\end{array}$ & $\begin{array}{l}\text { Unum }=500 \text { units/day, } \\
\text { maximum }=1,500 \text { units/day }\end{array}$ & $\begin{array}{l}\text { Exponential distri- } \\
\text { bution, mean }= \\
1.000 \text { units/day }\end{array}$ \\
$C_{d}$ & Non correlated observations & $\begin{array}{l}\text { Slightly correlated, first 4-5 } \\
\text { lags correlated }\end{array}$ & Highly correlated \\
& & Mean =7 days & Mean $=9$ days \\
$\mu_{L T}$ & Mean = 5 days & $\mathrm{CV}=0.1$ & $\mathrm{CV}=0.2$ \\
$\sigma_{L T}$ & $\mathrm{CV}=0$ & & \\
\hline
\end{tabular}

All lead times, expect the constant values, are normal i.i.d.. The Coefficient of Variance $(\mathrm{CV})$ is used to scale the variation in lead times defined as:

$$
C V=\frac{\sigma_{L T}}{\mu_{L T}} \Rightarrow \sigma_{L T}=C V \times \mu_{L T}
$$

Where $\mu_{\mathrm{LT}}$ is the mean of the lead time and $\sigma_{\mathrm{LT}}$ is the standard deviation, so for e.g. $\mu_{\mathrm{LT}}=7$ days, $\mathrm{CV}=0.2$ the standard deviation used is $0.2 \times 7$ days $=1.4$ days. All demand distributions have a mean of 1.000 units/day for both independent and dependent distributions. In the case of this experiment all factors are treated as categorical factors. This means that a linear response is not expected. It also means that it will be possible to identify not just which factors are significant for any of the four performance measures (responses) but also at which levels they are critical. This will enable a discussion of which factors should be taken into account when using a Re-orderpoint inventory management method in practice.

The four performance measures investigated in this paper are all service level measures. They are defined as follows:

SL1: calculated as the ratio between stock out in pieces and total demand.

SL2: calculated as the ratio between stock out in days (the sum of days when a stock out occurred) and total number of days. 
SL3: calculated as the ratio between stock out in pieces and total demand during the period while at least one order is open.

SL4: calculated as 1 minus the ratio between the number of "stock out" and the total number of orders. A "stock out" is considered to have occurred when the total demand in a lead time exceeds the mean demand over the lead time.

The simulation model was developed according with the standard EOQ model for single item. A set of stochastic functions, developed in the SciLab environment, are used to generate the demand that activates the model. The simulations are conducted for a length of 1.000 days to guarantee a stable output and thus a correct estimation of the four service level measures.

\section{$4 \quad$ Analysis of Results}

The design and analysis of experiments have been conducted using the open source software R (r-project.org, 2012) and the package AlgDesign. The ANOVA tables for all four fitted models are presented in Table 2. The general investigation of the fitted response models show the following: QQ-plots indicate normal distributed residuals for all four models indicating a reasonable fit of models. Adjusted R2 are in the interval 0.83 to 0.90 likewise indicating a good fit of all the models. This strongly indicates that the models are valid descriptors of the behavior of the four performance measures investigated in this study.

\subsection{Service Level}

Table 2 shows the ANOVA of the four service level models for the models. The table includes coefficients and p-values. The values shown in Table 2 indicate firstly the coefficients and their corresponding p-values. The coefficients indicates the response difference from the baseline model where all factors assume the value -1 . This means it is possible to see when there is a significant effect of a factor or combination of factors at given values. This makes it possible not only to identify which factors are significant but also on which settings they are critical.

The values shown in Table 2 indicate that there are some generic responses to given settings of the factors, furthermore it is also apparent that all four response models have significant (on a 0.05 or better level) second order interactions. This illustrates the complexity faced when doing real life inventory management, as second order interactions must apparently be considered when designing and implementing inventory management. The $\mathrm{R}^{2}$ for the four models are respectively for SL1-4: 0.97, 0.97, 0.98 and 0.97 and adjusted $\mathrm{R}^{2}$ are: $0.87,0.84,0.9$ and 0.83 . The high values of both $\mathrm{R}^{2}$ and adjusted $\mathrm{R}^{2}$ indicate a very good model fit, which is of course to be expected when all the parameters are included. The results of the factorial analysis will be split in to three parts. The first will focus on the factors and combination of factors and settings that are critical for all four service level measures. The second will focus on the individual service levels and the factors that are significant only for this particular measure. The third will focus on response models only containing main effects. 
Table 2. p-values from ANOVA for SL1, SL2, SL3 and SL4 and coefficients of the fitted models. p-values emphasized with bold indicate variables significant on a better than 0.05 level. To improve readability only rows containing significant variables have been included.

\begin{tabular}{|c|c|c|c|c|c|c|c|c|}
\hline \multirow[b]{3}{*}{ (Intercept) } & \multicolumn{2}{|c|}{ SL1 } & \multicolumn{2}{|c|}{ SL2 } & \multicolumn{2}{|c|}{ SL3 } & \multicolumn{2}{|c|}{ SL4 } \\
\hline & Estimate & $\operatorname{Pr}(>|t|)$ & Estimate & $\operatorname{Pr}(>|t|)$ & Estimate & $\operatorname{Pr}(>|t|)$ & Estimate & $\operatorname{Pr}(>|t|)$ \\
\hline & 0.999 & 0.000 & 0.998 & 0.000 & 0.999 & 0.000 & 0.983 & 0.000 \\
\hline$D_{d} l$ & -0.012 & 0.011 & -0.012 & 0.003 & -0.017 & 0.002 & -0.047 & 0.003 \\
\hline$D_{d} l: C_{d} l$ & -0.009 & 0.099 & 0.004 & 0.398 & -0.016 & 0.017 & 0.011 & 0.538 \\
\hline$D_{d} l: \mu_{L T} l$ & -0.012 & 0.036 & -0.007 & 0.097 & -0.008 & 0.203 & -0.025 & 0.167 \\
\hline$D_{d} l: C_{d} 1: \mu_{L T} 0$ & 0.012 & 0.042 & 0.002 & 0.610 & 0.019 & 0.008 & -0.013 & 0.487 \\
\hline$D_{d} 1: C_{d} 0: \mu_{L T} 1$ & 0.016 & 0.012 & 0.014 & 0.008 & 0.021 & 0.005 & 0.063 & 0.004 \\
\hline$D_{d} l: C_{d} l: \mu_{L T} l$ & 0.012 & 0.042 & 0.003 & 0.513 & 0.020 & 0.007 & 0.018 & 0.351 \\
\hline$D_{d} 1: C_{d} 1: \sigma_{L T} 0$ & -0.015 & 0.016 & -0.012 & 0.014 & -0.019 & 0.009 & -0.047 & 0.023 \\
\hline$D_{d} l: C_{d} 0: \sigma_{L T} l$ & -0.011 & 0.066 & -0.009 & 0.062 & -0.014 & 0.044 & -0.021 & 0.287 \\
\hline$D_{d} 1: C_{d} 1: \sigma_{L T} 1$ & -0.012 & 0.042 & -0.011 & 0.022 & -0.015 & $\mathbf{0 . 0 3 3}$ & -0.030 & 0.125 \\
\hline
\end{tabular}

In general all four SL measures are only sensitive to one main effect and that is $D_{d}$ and only at the setting of 1 . In all four cases the coefficient is negative for this setting of $D_{d}$, and in all cases is the setting of 0 in no way significant. Both the size of the coefficient and the significance level the demand distribution setting of 1 - indicating an asymmetrical distribution - is the largest for all four models. This strongly indicates that the asymmetrical demand distribution (supported by the fact that the other symmetrical distribution has no significant different response than the base line setting -1) is the single most significant factor in explaining lower service levels. It also strongly indicates that the re-order point method is highly sensitive to shape of the demand distribution, especially when it is skewed. Previous work by e.g. Eriksen and Nielsen (2011) and Nielsen et al. 2010 indicates that demand rates are in fact neither normal nor symmetrical distributed in practice. This underlines that this topic deserves further investigations.

Another generic significant effect is the second order interaction $\mathrm{D}_{\mathrm{d}} @ 1: \mathrm{C}_{\mathrm{d}} @ 0: \mu_{\mathrm{LT}} @ 1$, indicating that demand is asymmetrically distributed, slightly correlated and lead time is long. In all four response models this combination of settings tends to increase service levels. The reason for the increase of service levels when three of the factors have these settings must be found in the central limit theorem. Under long lead times $\left(\mu_{\mathrm{LT}} @ 1\right)$ the asymmetrical distributed demand $\left(\mathrm{D}_{\mathrm{d}} @ 1\right)$ will tend towards a normal distribution with low variation. However, it is interesting to note that $\mu_{\mathrm{LT}} @ 1$ is not significant as a main effect, most likely because this is covered in safety stocks and calculation of these levels. A conclusion must be that to avoid the detrimental effects of a skewed demand distribution (i.e. $D_{d} @ 1$ ) on service levels practitioners must compensate by aggregating in time (See e.g. Fliedner (2001)). This finds support in Nielsen et al. (2010) and methods for calculating 
adequate aggregation horizons to achieve a satisfactory distribution of demand can be found in Eriksen and Nielsen (2011).

$\mathrm{D}_{\mathrm{d}} @ 1: \mathrm{C}_{\mathrm{d}} @ 1: \sigma_{\mathrm{LT}} @ 0$ is likewise a significant second order interaction with a negative contribution in all four models. This combination of factors is for a dependently asymmetrical distributed demand with a low variation in lead time. The combination of the first two factors is not surprising as this would tend towards giving a very skewed demand distribution. However, it is difficult to establish why slightly nonconstant lead time should be is significant.

Other noteworthy factors and interactions is $D_{d} @ 1: C_{d} @ 1: \sigma_{L T} @ 1$ that has a significant negative impact on SL1-3. This second order interaction actually indicates the almost worst case scenario. Highly correlated, asymmetrically disturbed demand, with large variation in lead times will naturally tend to lead to a lower service level. It is in fact interesting why it is not also a significant combination of factors for the fourth SL measure.

Several generic conclusions can be reached from this study. First, that asymmetrical demand distributions tend to lead to lower service levels. This is interesting since several studies of real life demand indicate that the demand faced by companies can in fact be asymmetrically distributed and also correlated in time. This also supports the findings of e.g. Zotteri (2000). Second, that to compensate for this one has to aggregate in time, in the case of inventory management this is typically achieved through longer lead times. This of course will tend to lead to higher holding costs, so here the trade-off between service and costs become critical in practice. This area deserves further study. A general problem with a full scale model with second order interactions is that there is a risk of over fitting. For this reason it could be prudent to investigate the reduced response models only including the main effects. An over view of these response models can be seen in Table 3 below.

Interestingly enough also when only main effects are considered the adjusted $\mathrm{R}^{2}$ only drops to the range $0.60-0.78$, which indicates a reasonable response model but with less precision. However, these models are interesting as they illustrate service level issues in a parameter by parameter manner. It is interesting to note that $D_{d} @ 1$ is still the single most critical factor, and that this setting reduces service level measures in all response models. It is also interesting to note that for three out of the four measures $\mathrm{C}_{\mathrm{d}} @ 1$ leads to a lower service level. The same goes for $\sigma_{\mathrm{LT}} @ 1$ although the contribution to the service levels are for all models (SL4 exempt) quite low. It is also interesting to note that only two out of $4 \times 4$ factors set at 0 are in fact significant. Of these only $\mu_{\mathrm{LT}} @ 0$ for SL4 has any significant contribution to the service level measure. This underlines that a large deviation from the assumed behavior is necessary for it to significantly affect service level performance. The combined impression is that performance primarily depends on whether or not a skewed demand distribution is present, and to some extend whether demand is highly correlated. The conclusion is again that the demand behavior is critical for the service level. A highly correlated demand will also tend to lead to demand being asymmetrically distributed in the short term, again underlying that the asymmetry of demand distributions is in fact highly 
critical for service level performance, regardless of which SL measure is used. It is also interesting to note from Table 3 that all deviations from the standard setting of the factors to -1 tend to lead to a negative response on all four SL measures.

Table 3. p-values from ANOVA for SL1, SL2, SL3 and SL4 and coefficients for the fitted response models only including main effects. P-values emphasized with bold indicate variables significant on a better than 0.05 level.

\begin{tabular}{lllllllll} 
& \multicolumn{3}{c}{ SL1 } & \multicolumn{2}{c}{ SL2 } & \multicolumn{2}{c}{ SL3 } & \multicolumn{2}{c}{ SL4 } \\
\cline { 2 - 9 } & Estimate & $\operatorname{Pr}(>|t|)$ & Estimate & $\operatorname{Pr}(>|t|)$ & Estimate & $\operatorname{Pr}(>|t|)$ & Estimate & $\operatorname{Pr}(>|t|)$ \\
\cline { 2 - 9 } (Intercept) & 1.002 & 0.000 & 0.999 & 0.000 & 1.001 & 0.000 & 0.990 & 0.000 \\
$D_{d} 0$ & 0.000 & 0.829 & 0.000 & 0.737 & 0.000 & 0.838 & -0.001 & 0.797 \\
$D_{d} l$ & $\mathbf{- 0 . 0 1 7}$ & $\mathbf{0 . 0 0 0}$ & $\mathbf{- 0 . 0 1 1}$ & $\mathbf{0 . 0 0 0}$ & $\mathbf{- 0 . 0 2 3}$ & $\mathbf{0 . 0 0 0}$ & $\mathbf{- 0 . 0 4 3}$ & $\mathbf{0 . 0 0 0}$ \\
$C_{d} 0$ & -0.002 & 0.158 & -0.001 & 0.256 & -0.003 & 0.126 & -0.008 & 0.110 \\
$C_{d} l$ & $\mathbf{- 0 . 0 0 4}$ & $\mathbf{0 . 0 0 1}$ & -0.002 & 0.073 & $\mathbf{- 0 . 0 0 6}$ & $\mathbf{0 . 0 0 1}$ & $\mathbf{- 0 . 0 1 3}$ & $\mathbf{0 . 0 0 6}$ \\
$\mu_{L T} 0$ & -0.002 & 0.187 & $\mathbf{- 0 . 0 0 3}$ & $\mathbf{0 . 0 1 8}$ & 0.001 & 0.556 & $\mathbf{- 0 . 0 1 0}$ & $\mathbf{0 . 0 3 8}$ \\
$\mu_{L T} l$ & -0.001 & 0.310 & -0.001 & 0.217 & 0.002 & 0.137 & -0.003 & 0.544 \\
$\sigma_{L T} 0$ & -0.001 & 0.255 & 0.000 & 0.663 & -0.002 & 0.249 & 0.000 & 0.981 \\
$\sigma_{L T} 1$ & $\mathbf{- 0 . 0 0 3}$ & $\mathbf{0 . 0 1 1}$ & $\mathbf{- 0 . 0 0 3}$ & $\mathbf{0 . 0 0 5}$ & $\mathbf{- 0 . 0 0 4}$ & $\mathbf{0 . 0 1 5}$ & $\mathbf{- 0 . 0 1 2}$ & $\mathbf{0 . 0 1 2}$ \\
\hline
\end{tabular}

\section{Conclusion}

The aim of the study has been to investigate which parameters are critical for a given performance criteria of a re-order point inventory model. The conclusion is that there is a significant difference in the importance of the four investigated factors depending on the performance measure. From this study it can conclusively be stated that skewed demand distribution is the single most detrimental factor with regards to service level performance. It can also be concluded that there are several second order interactions that are highly significant, underlining the fact that inventory management is in fact a highly complex problem. Furthermore the findings of the presented investigations support the conclusions reached in e.g. Tadikamalla (1984) and Zotteri (2000), namely that the asymmetry of demand distributions are in fact highly significant for the performance of inventory management methods. Based on the study it is possible to give direct conclusive guidelines for where to focus when improving service levels in real-life applications of the ROP method. Especially it should be noted that the only feasible manner to compensate for the negative effects of asymmetrically distributed demand is to aggregate in time, through a longer safety period.

The conclusion must necessarily be that the complexity of inventory management is high, since the performance of a given ROP managed system cannot be predicted solely based on the main effects affecting it. This underlines that research into the behavior of inventory management systems and their performance is in fact a topic of relevance for both researchers and practitioners. 


\section{References}

1. Bobko, P.B., Whybark, D.C.: The Coefficient of Variation as a Factor in MRP Research. Decision Sciences 16(4), 420-427 (1985)

2. Brander, P., Levén, E., Segerstedt, A.: Lot sizes in a capacity constrained facility-a simulation study of stationary stochastic demand. International Journal of Production Economics 93-94, 375-386 (2005)

3. Eriksen, P.S., Nielsen, P.: Emperical order quantity distributions: At what level of aggregation do they respect standard assumptions? In: Proceedings of the International Conference on Advances in Production Management Systems, University of Stavanger (2011)

4. Fliedner, G.: Hierarchical forecasting: issues and use guidelines. Industrial Management \& Data Systems 101, 5-12 (2001)

5. Lau, H.-S., Lau, A.H.-L.: Nonrobustness of the Normal Approximation of Lead-Time Demand in a (Q, R) System. Naval Research Logistics 50, 149-166 (2003)

6. Nielsen, P., Nielsen, I., Steger-Jensen, K.: Analyzing and Evaluating Product Demand Interdependencies. Computers in Industry 61(9), 869-876 (2010)

7. Pujawan, I.N., Kingsman, B.G.: Properties of lot-sizing rules under lumpy demand. International Journal of Production Economics 81-82, 295-307 (2003)

8. Silver, E.A., Pyke, D.F., Peterson, R.: Inventory Management and Production Planning and Scheduling, 3rd edn. John Wiley \& Sons (1998)

9. Tadikamalla, P.R.: A Comparison of Several Approximations to the Lead Time Demand Distribution. Omega 12, 575-581 (1984)

10. Vollmann, W., Berry, D., Whybark, T.E., Jacobs, F.: Manufacturing Planning and Control for Supply Chain Management. McGraw-Hill, Singapore (2005)

11. Wijngaard, J.: On Aggregation in Production Planning. Engineering Costs and Production Economics 6, 259-266 (1982)

12. Zotteri, G.: The impact of distributions of uncertain lumpy demand on inventories. Production Planning \& Control 11, 32-43 (2000) 